\title{
Effect of the Duration Time of the Action Sentence Representation on the Judgment of the Present Time*
}

\author{
Zhang Shilei, Ba Xiaona, Xu Peng \\ Huihui College of Hebei Normal University, \\ Shi Jiazhuang, P.R. China
}

\author{
Lu Zhongyi \\ Hebei Normal University, \\ Shi Jiazhuang, P.R. China
}

\begin{abstract}
Objective: Embodied cognitive theory holds that the process of language comprehension is a process of metal simulation. Verbal comprehension is a simulation of the action and completing the action needs time which affects the judgment of presentation time of verbs. In this study, participants are asked to read the sentences which are different in the presentation time. The presentation time of experimental sentences is $4000 \mathrm{~ms}$ and the filling sentences are $2000 \mathrm{~ms}, 3000 \mathrm{~ms}, 5000 \mathrm{~ms}, 6000 \mathrm{~ms}$ or $7000 \mathrm{~ms}$ randomly. The duration time of the action which the experimental sentences represent is divided into two types. After reading the sentence, it is necessary to determine the present time of the sentence. Finding that the during time of the action is long and the present time of the sentence is long by participants judged; when the during time of the action is short, the present time of the sentence is short by participants judged.

Keywords: embodied, verb, duration time, judgment
\end{abstract}

\section{Introduction}

Many studies claim embodied cognitive theory can explain many results of the language understanding study better than the traditional view (Willems \& Casasanto, 2011; Zwaan, 2004, 2014). The traditional view treat language as a symbol system which is abstract, amodal, and arbitrary (Kintsch, 1988; Pinker, 1994), so language comprehension is an advanced process, not involving perception and movement system, for example the concept of language representation should not involve the concrete form of the object. But embodied cognitive theory holds that language understanding involves the biomechanical nature of bodies and perceptual systems (Glenberg, 1997; Lakoff, 1987). In other words, the sensorimotor system is activated in the processing of language. The activated perceptual representations are the building blocks of cognition making up cognitive processes and they are the residues of a perceptual experience (Barsalou, 1999). Understanding words and sentences involves the reactivation of perceptual experiences (Zwaan, 2004). When the shape of the object which implied by the sentences changed, language comprehenders would represent the implied shape (Zwaan, Stanfield, \& Yaxley, 2002); reading words that denote actions can also reactive the sensorimotor system (Hauk, Johnsrude, \& Pulvermüller, 2004). The format of concepts is embodiment and not abstract, amodal, or

\footnotetext{
*Acknowledgments: This work was supported by Huihua College of Hebei Normal University.

Zhang Shilei, Lecturer, Huihua College of Hebei Normal University.

Ba Xiaona, Lecturer, Huihua College of Hebei Normal University.

Xu Peng, Lecturer, Huihua College of Hebei Normal University.

Lu Zhongyi (Corresponding Author), Professor, Faculty of Education, Hebei Normal University.
} 
symbolic (Glenberg, 2015; Mahon, 2015), abstract language grounded in bodily experiences of perception and action (Glenberg, Sato, Cattaneo, Riggio, Palumbo, \& Buccino, 2008).

A lot of researches on verb comprehension also prove the embodiment of language understanding. Understanding verb activates different effector organs. The verb to represent the action of the mouth can activate the perceptual motor system relating mouth action and the perceptual motor system involving foot moving is active when read the verb representing foot action (Scorolli \& Borghi, 2007). It shows that the process of verb comprehension involves mental simulation and the simulation is different because the effector is different. Some studies show that comprehension of the action sentences may involve motor resonance. Participants who listened to sentences which describe an action close or far away from body judge the meaning of the sentences by pressing a button which required a movement toward or away from themselves. It shows an action-compatibility effect (ACE), when the movement direction implied by sentences was the same as the physical response, participants response were faster (Glenberg \& Kaschak, 2002). At the same time, there is ACE in the understanding of abstract verbs describing information transmission (Glenberg et al., 2008). Understanding language representing concrete and abstract events involves the motor system and use-induced plasticity at the level of action, not just at the level of motor control (Glenberg, Sato, \& Cattaneo, 2008). Above the research, the movement direction of the sentence representation is horizontal; when the movement direction was to rotate, there was an action-compatibility effect (ACE). Participants listened to recordings of sentences described manual rotation and then made sensibility judgments about them. They turned the knob either to the right for a yes response and to the left for a no response or the other way around, founding that manual response faster when it was congruent with the action described in a sentence than incongruent responses (Zwaan \& Taylor, 2006). In addition, the movement direction in other words the spatial representation of the verb affects the visual processing tasks in the same direction. Participants who listened to sentences which include the transverse or longitudinal spatial representation of verbs judge the shape of the graphics that presented the horizontal or vertical direction of the screen. When the spatial representation of verbs was the same as the position of graphics, the reaction was slow. This result demonstrated understanding verbs activated spatial representation (Richardson, Spivey, Barsalou, \& McRae, 2003). These empirical finding indicates verbal comprehension involves metal simulation and these simulations involve the action of effector, movement direction, etc.. Verb comprehension is like real action acting. In everyday life, it takes time to do anything. Some things need a long time and other things need a short time. Is the experience of these time perception as the direction of movement during in the process of understanding the verb? Or does the simulation involve the duration of the action? If it is true, we think it should influence the judgment of the sentence presentation time just as verbs understand impacted the perceptual processing in motion direction. When the execution action which the sentence represents takes a longer time than others, participant will estimate the duration time of the sentence longer, in fact the duration time of the sentence is the same. When the execution action which the sentence represents takes a shorter time than others, participant will estimate the duration time of the sentence shorter, but the duration time of the sentence is the same. This study is to verify the hypothesis.

\section{Method}

\section{Participants}

Thirty students participated in the experiment from Huihua College of Hebei Normal University, who studied the mental health courses. All students were girls. They receive course credit for their participation. 


\section{Materials}

Through the questionnaire, the researchers assessed the experimental sentences. First, 26 groups of sentences were prepared, the two sentence in one group were the same in the subject, structure and number of words. Second, 20 college students estimated the time required for the completion of the 52 sentences described action in seconds. These students did participate in the formal experiment. The last, 10 groups of sentences were chosen as experimental materials, there were statistically significant differences in the completion time between the sentences in each group, for example "I wrote a word" and "I draw a picture". According to the duration of the action, the experimental sentences were divided into two groups, which contained ten sentences. In addition, there were 6 groups of sentences as a practice and 16 groups of sentences as filling materials. The materials were in Chinese.

\section{Procedure}

The experiment was done in a quiet laboratory, and the experiment was carried out by E-Prime 2.0. Participants were tested on a computer screen with a distance of about 60 centimeters, 17 inches. On the screen, the instruction was presented, and then entered the experimental program. Before the start of the formal experiment, 12 exercise sentences helped to be familiar with the test procedure. After practice, participants were still not familiar with the experimental procedures, can be through the "Q" button to repeat the exercise, if already familiar with, press "P" key to enter the formal experiment. The trials were like this. First, a "+" appeared in the center of the screen, during 500ms. Second, a sentence presented, participants should understand the sentence. When the sentence was experimental, the present time was 4000ms; the sentence was filled, the during time was $2000 \mathrm{~ms}, 3000 \mathrm{~ms}, 5000 \mathrm{~ms}, 6000 \mathrm{~ms}$ or $7000 \mathrm{~ms}$. After the sentence participants tried to press the space bar twice, required to press the time interval of the space keys to be consistent with the presentation time of the previous sentence and entered the specific time that the sentence was presented by pressing the number key. At last, they pressed enter key to appear next "+", the experiment continued until the end. The whole experiment took about ten minutes.

\section{Results}

One participant did not understand the requirements of the experiment, so didn't statistics, the others were statistically analyzed.

The interest of this experiment was to determine whether there was a difference in judgment of the time of sentence presentation, including two forms: one was the interval time of the pressed space key; the other was the input time. Repeated measurement ANOVA to analyze on the interval time was significant, $F(1,28)=4.73$, $p<0.05$; Repeated measurement ANOVA to analyze on the input time was significant, $F(1,28)=4.73, p<0.05$. These results indicated that after understanding the sentence which described the action completion time longer, presented time of the sentence was judged to be longer; on the contrary can also be set up. Paired $t$-test conducted on the interval time was significant, $t(9)=3.30, p<0.05$; Paired $t$-test conducted on the input time was significant, $t(9)=5.62, p<0.05$. The execution time of a sentence was shorter, the presentation time was shorter by participants judged; the opposite was the same.

\section{Discussion}

The experimental results conform the research hypothesis that verb comprehension activates the time to complete action which verb represents. The finding is not in accordance with the traditional view, because the 
traditional view held that language comprehension was advanced processing, having no necessary connection with the sensorimotor system. In the present study, the presentation time of all the experimental sentences was the same, but there were significant differences in the judgment of participants. These judgments are consistent with the actual completion time of the action. The understanding of verbs is closely related to our own experience and the time perception experience affected the judgment of the time of sentence presentation. It matches the embodied cognitive theory which indicated the language comprehension is embodiment. In recent years, many empirical studies have found that there is a close relationship between language and the sensorimotor system. Previous research had shown that the processing of action verbs elicits automatic activation of the cortical motor systems in a somatotopic manner (for a review, see Kiefer \& Pulvermüller, 2012). This hypothesis was also supported by behavioral studies (Dalla, Gianelli, Campione, \& Gentilucci, 2009; Sato et al., 2008). Reading words that token actions also activated the motor system (Hauk, Johnsrude, \& Pulvermüller, 2004). Repeated grasping and moving of objects from a position near to the body to a position far from the body affects judgments about sentences referring to actions (Glenberg \& Kaschak, 2002; Glenberg et al., 2008). As mentioned before, the study of sentence comprehension had found the ACE. These studies have indicated that the verb comprehension is a metal simulation process of the action which the verb represents. This simulation process is similar to real execution of the action. The completion of each action will require a certain time, the simulation process involves the during time of the action. The study proves it.

From the perspective of time, it was further proved that the understanding of verbs was a mental simulation of the real action, because the processing of the verb included not only the movement direction of the real movement, but also the duration time of the action. Just as spatial representation of verbs affecting the judgment of the graphics shape, the during time of verbs affected the judgment of present time. Through the behavioral experiment, it proved that the verb understand involves performing time of an action. In the future, it may be shown that action understanding involved brain areas which associate time perception through the neurophysiological method.

In daily life, people do things, not only involves the movement direction, action time, but also contains their own emotions, these emotions affect people's perception of time. For example, when doing things spend the same time, happy people perceive time flying and anxious people feel time slowing in our life. Moreover, it had been found that language comprehension can cause people's emotional reaction (Niedenthal, Winkielman, Mondillo, \& Vermeulen, 2009), whether the emotion activated by language would also impact the time perception of the language itself like daily life?

\section{References}

Barsalou, L. W. (1999). Perceptual symbol systems. Behavioral and Brain Sciences, 22, 577-660

Dalla, R., Gianelli, C., Campione, G. C., \& Gentilucci, M. (2009). Action word understanding and overt motor behavior. Exp. Brain Res., 196, 403-412.

Glenberg, A. M. (1997). What memory is for. Behavioral and Brain Sciences, 20, 1-55.

Glenberg, A. M. (2015). Few believe the world is flat: How embodiment is changing the scientific understanding of cognition. Canadian Journal of Experimental Psychology, 69, 165-171.

Glenberg, A. M., \& Kaschak, M. P. (2002). Grounding language in action. Psychonomic Bulletin \& Review, 9, 558-565.

Glenberg, A. M., Sato, M., \& Cattaneo, L. (2008). Use-induced motor plasticity affects the processing of abstract and concrete language. Current Biology, 18, R290-R291. Retrieved from http://dx.doi.org/10.1016/j.cub.2008.02.036

Glenberg, A. M., Sato, M., Cattaneo, L., Riggio, L., Palumbo, D., \& Buccino, G. (2008). Processing abstract language modulates motor system activity. The Quarterly Journal of Experimental Psychology, 61(6), 905-919. 
Hauk, O., Johnsrude, I., \& Pulvermüller, F. (2004). Somatotopic represen-tation of action words in human motor and premotor cortex. Neuron, 41, 301-307. http://dx.doi.org/10.1016/S0896-6273(03)00838-9

Kiefer, M., \& Pulvermüller, F. (2012) Conceptual representations in mind and brain: Theoretical developments, current evidence and future directions. Cortex, 48, 805-825. doi:10.1016/j.cortex.2011.04.006

Kintsch, W. (1988). The role of knowledge in discourse comprehension: A construction-integration model. Psychological Review, 95,163-182.

Lakoff, G. (1987). Women, fire, and dangerous things: What categories reveal about the mind. Chicago: University of Chicago Press.

Mahon, B. Z. (2015). What is embodied about cognition? Language, cognition, and neuroscience. Retrieve from https://caoslab. bcs.rochester.edu/pdf/Mahon_InPress_LCN.pdf. doi:10.1080/23273798.2014.987791

Niedenthal, P. M., Winkielman, P., Mondillon, L., \& Vermeulen, N. (2009). Embodiment of emotion concepts. Journal of Personality and Social Psychology, 96(6), 1120-1136.

Pinker, S. (1994). The language instinct. New York: Harper Collins.

Richardson, D. C., Spivey, M. J., Barsalou, L. W., \& McRae, K. (2003). Spatial representations activated during real-time comprehension of verbs. Cognitive Sciences, 27(5), 767-780.

Sato, M., Mengarelli, M., Riggio, L., Gallese, V., \& Buccino, G. (2008). Task related modulation of the motor system during language processing. Brain Lang, 105(2), 83-90.

Scorolli, C., \& Borghi, A. (2007). Sentence comprehension and action: Effector specific modulation of the motor system. Brain Research, 1130, 119-124.

Willems, R. M., \& Casasanto, D. (2011). Flexibility in embodied language understanding. Frontiers in Psychology, 2, 116. doi: 10.3389 /fpsyg.2011.00116

Zwaan, R. A. (2004). The immersed experiencer: Toward an embodied theory of language comprehension. In B. H. Ross (Ed.), The psychology of learning and motivation (Vol. 44, pp. 35-62). New York, N.Y.: Academic Press.

Zwaan, R. A. (2014). Embodiment and language comprehension: Reframing the discussion. Trends in Cognitive Sciences, 18, 229-234. http://dx.doi.org/10.1016/j.tics.2014.02.008

Zwaan, R. A., Stanfield, R. A., \& Yaxley, R. H. (2002). Do language comprehenders routinely represent the shapes of objects? Psychological Science, 13, 168-171.

Zwaan, R. A., \& Taylor, L. J. (2006). Seeing, acting, understanding: Motor resonance in language comprehension. Journal of Experimental Psychology: General, 135, 1-11. 